\title{
Electrostatic precipitation pressurized intraperitoneal aerosol chemotherapy (ePIPAC): Finding the optimal electrical potential
}

\author{
Mohammad Rahimi-Gorji ${ }^{1,2,3}$, Charlotte Debbautt ${ }^{2,3}$, Ghader Ghorbaniasl ${ }^{4}$, Wouter Willaert ${ }^{1,3}$, Sarah \\ Cosyns $^{1}$, Wim Ceelen ${ }^{1,3}$ \\ ${ }^{1}$ Department of Human Structure and Repair, Ghent University, Ghent, Belgium \\ 2IBiTech- bioMMeda, Ghent University, Ghent, Belgium \\ ${ }^{3}$ CRIG- Cancer Research Institute Ghent, Belgium \\ ${ }^{4}$ Department of Mechanical Engineering, Vrije Universiteit Brussel, Brussels, Belgium
}

\section{BACKGROUND}

Pressurized IntraPeritoneal Aerosol Chemotherapy (PIPAC) is a therapy option for patients with peritoneal metastases. During PIPAC, a high-pressure injector delivers the drugs through a nebulizer into the peritoneal cavity. However, gravity typically causes inhomogeneous droplet distribution in the peritoneal space and may be overcome by the addition of an electrostatic force, resulting in electrostatic precipitation PIPAC (ePIPAC). Our goal was to find the optimal electrical potential for ePIPAC by means of in vitro experiments and numerical modeling.

\section{MATERIALS AND METHODS}

The ePIPAC in vitro experiments were performed in a Plexiglas box model, representing the peritoneal space. Rat peritoneal tissue samples $\left(185 \times 135 \times 152 \mathrm{~mm}^{3}\right)$ were placed on 4 plates mounted at the inner boundaries of the box. The box was first infused with $\mathrm{CO}_{2}$ gas to reach a pressure of $12 \mathrm{mmHg}$. Black ink $(20 \mathrm{~mL})$ was subsequently injected with a high-pressure injector (Injektron ${ }^{\mathrm{TM}}$, Germany) at a flow rate of $0.5 \mathrm{~mL} / \mathrm{s}$. Afterwards, the tissue specimens were digitally photographed and the distribution of ink was quantified using ImageJ. In addition, a computational fluid dynamics (CFD) model of the box was generated using the CFD, AC/DC and Particle Tracing Modules in COMSOL Multiphysics (Burlington, VT). The initial (zero pressure) and boundary conditions (inlet flow at $12 \mathrm{mmHg}$ ) were assumed, consisting of the pressure, flow rate and the properties of the ink with considering drag, gravitational and electrical forces. To study the impact of applied electrical potential, the electrostatic field was modeled using a brush electrode with a voltage of $4,5,6,6.5,7,8$ or $9 \mathrm{kV}$ as a source and four metal plates as grounds.

\section{RESULTS}

Imposing an electrostatic field to the PIPAC setup (ePIPAC) a more homogeneous aerosol droplet distribution in the box. A significant increase for proportion of ink (a minimum of $60 \%$ ) was observed at the stained tissue surface on the top wall of the box. The results showed an overall good agreement for the experiments and simulation. The electrical potential sensitivity analysis revealed that the aerosol droplets distribution became more homogeneous as the electrical potential increased, but no further improvements were obtained after $6.5 \mathrm{kV}$. Consequently, the optimum electrical potential for ePIPAC was $6.5 \mathrm{kV}$.

\section{CONCLUSION}

In conclusion, electrostatic precipitation was shown to improve drug distribution during PIPAC and, hence, may lead to better anticancer efficacy. Also, the simulation technique allows developing methods to predict droplet behavior and improve drug delivery. 